\title{
LOS DESAFÍOS DE FACEBOOK. Apuntes para el abordaje de las redes sociales como fuente
}

\author{
María Lorena CAPOGROSSI; \\ Mariana Loreta MAGALLANES; Florencia SORAIRE \\ CIECS-UE CONICET (Argentina) \\ lcapogrossi@yahoo.com.ar, loretmagas@gmail.com, antropoflor@gmail.com
}

THE CHALLENGES OF FACEBOOK. Notes for addressing social networks as a source

Resumen: Este artículo es un primer intento de sistematizar la utilización de las redes sociales como fuente en las investigaciones en ciencias sociales a partir de un estudio de caso. Nos proponemos aquí señalar las limitaciones y potencialidades que tiene específicamente Facebook, los retos a los que se enfrenta el investigador cuando se dispone a utilizarlo y los desafíos éticos que depara el uso de la información que los usuarios de las comunidades virtuales publican en la red. Entre los ejes que consideramos necesario problematizar podemos mencionar, por un lado, la fiabilidad y la mutabilidad de la información, que son características intrínsecas del recurso técnico utilizado, y por el otro, la puesta en tensión de la idea de territorialidad y de la presencia física del investigador en el trabajo de campo. Estos aspectos de la cuestión generaron numerosos debates que derivaron en esta propuesta de sistematización que abre un abanico de preguntas que esperamos poder profundizar a partir de este análisis.

Abstract: This paper is a first attempt to systematize the use of social networks as source in the social science research, from a case study. This proposal have like objective to observe the limitations and potentials that have the social networks, specifically Facebook, when they are used in social researches. We propose analyse the challenges facing the researcher when working with this type of source. At the same time, we'd like to problematize the ethical challenges arising from the use made of the information that users post on the social network. Among the axes that we consider necessary to analyze we can mention, on the one hand, the reliability and the mutability of information. Both are inherent characteristics of the technical device used. Secondly, we seek to question the idea of territoriality and the idea of physical presence of researcher in the field. These aspects of the question have generated numerous debates. Here, we will systematize a series of questions generated from these discussions.

Palabras clave: Redes sociales. Fuentes de Información. Investigación Académica. Desafíos técnicos, legales y éticos.

Social Networking. Sources of information. Academic research. Technical, legal, ethical challenges 


\section{Introducción}

Un aspecto fundamental en la construcción de toda trayectoria de investigación lo constituye la selección y el abordaje de las fuentes. En la actualidad, las posibilidades que brinda Internet respecto al acceso a la información son múltiples. Por un lado, se diversifican las temáticas y las metodologías de trabajo y, por el otro, la información circula con una inmediatez que posibilita seguir determinados acontecimientos prácticamente en tiempo real.

Es necesario señalar, sin embargo, que la utilización de estos recursos virtuales en los procesos de construcción del conocimiento, obliga a realizar ciertos recaudos y establecer algunas precisiones. Entre ellos, el problema de la fiabilidad de lo que se construye o se dice en esos espacios virtuales es un dato no menor que debe ser pensado, así como la mutabilidad de la información, de lo que está en un momento y desaparece después, teniendo en cuenta que todo lo que se publica puede ser eliminado por su autor. En ese sentido, reflexionar acerca del uso de las redes sociales como fuente, abre un espacio reflexivo que es interesante sistematizar.

El abordaje de comunidades virtuales fue una inquietud que surgió a partir de una investigación mayor, donde el objeto de estudio eran las prácticas y memorias de un colectivo de trabajadores de la empresa estatal Yacimientos Petrolíferos Fiscales. Allí se utilizaron, como parte del acervo documental, aquellas publicaciones que los ex petroleros consignaban en un grupo de Facebook. En esta ocasión, nos proponemos realizar una primera aproximación al análisis de las redes sociales, particularmente a su utilización como fuente. Al mismo tiempo, reseñaremos cómo las hemos abordado y qué preguntas nos atravesaron al momento de sistematizarlas. No pretendemos exhaustividad en este trabajo, sino comenzar a puntualizar las potencialidades y limitaciones que tienen estos recursos en las investigaciones en ciencias sociales.

A partir de la metodología que formó parte de la tesis doctoral ${ }^{1}$ de una de nosotras, recuperamos la iniciativa de trabajar de manera conjunta la problemática de las redes sociales y, en particular, de Facebook. La utilización de esta última en la investigación que aquí hemos tomado como punto de partida, surgió de la necesidad de encontrar otras maneras de acercarse a los informantes. El universo de entrevistados estaba constituido por ex trabajadores petroleros y sus familias y la característica que compartían era la de haber vivido por décadas en un campamento petrolero de la provincia de Salta, al norte de Argentina, bajo la órbita de una empresa estatal, Yacimientos Petrolíferos Fiscales (YPF). Muchos de esos actores, cuando fue privatizada la empresa en 1992, tuvieron que migrar a otras regiones. Este proceso supuso tener que rastrear a esos ypefianos ${ }^{2}$ fuera de aquel territorio, donde a lo largo de las décadas habían construido sus relaciones comunitarias.

Luego de acercarnos al campo, a la comunidad de Campamento Vespucio, observamos que las relaciones propias de la vida en la villa obrera habían sido desarticuladas desde la privatización de YPF debido a los conflictos laborales y las migraciones de población que se produjeron. Sin embargo, logramos identificar diferentes canales que esos sujetos encontraron para continuar vinculándose, para mantenerse en contacto e interactuar, pese a no ser ya vecinos. Consideramos entonces que era posible abordar esas otras maneras de reconstituir las relaciones $\mathrm{y}$, de esta manera, llegamos a una comunidad virtual en www. facebook.com. Pensarla como fuente supuso tensionar la idea de presencialidad física del

\footnotetext{
1 Capogrossi, María Lorena (2013): La relación capital-trabajo en «economías de enclave» y sus transformaciones durante el proceso de ajuste estructural en los 90. El caso de los trabajadores de YPF en Campamento Vespucio y General Mosconi, Salta". Tesis doctoral. Doctorado de la Universidad de Buenos Aires, orientación Antropología. Área de Posgrado. Facultad de Filosofía y Letras. Universidad de Buenos Aires. Inédita.

2 Ypefianos es una categoría nativa. De esa manera se autodefinen los ex trabajadores petroleros que estuvieron vinculados a YPF
} 
investigador en el campo - tan asociada al hacer etnografías - y asumir el desafío de afrontar nuevas experiencias metodológicas y problematizaciones derivadas de su utilización y de su legitimidad.

\section{El universo Facebook}

Facebook es una de las redes sociales más grandes que existen en la web en la actualidad, la cifra de usuarios para el año 2013 está estimada en 1.000 millones de personas en el mundo. Esta red fue creada en 2004 por un estudiante de la Universidad de Harvard llamado Mark Zuckerberg, con el objetivo de que los alumnos de esa universidad pudiesen interconectarse entre sí a través de la creación de un perfil donde, además de sus datos personales, podían incorporar fotos, generar foros de discusión, etc. Con el tiempo se extendió a otras universidades y unos años después se convirtió en un fenómeno generalizado al cual se podía acceder teniendo una cuenta de correo electrónico. Definido por sus ejecutores desde lo normativo, señalan que su misión es:

“dar a la gente el poder de compartir y hacer el mundo más abierto y conectado... Estamos construyendo Facebook para lograr un mundo más abierto y transparente, con lo que creemos que se alcanzará un mayor entendimiento y conexión. Facebook promueve la sinceridad y la transparencia ofreciendo a los individuos un mayor poder para comunicarse y compartir" (http://www. facebook.com/facebook\#!/facebook?sk=info0. Acceso 4/02/2004).

Ciertos pilares guían a Facebook en la persecución de estos objetivos, para ello sus creadores se basan en principios básicos que determinan lo que las personas pueden hacer, entre ellos:

“(...) disfrutar de la libertad de compartir cualquier información, a través de cualquier medio y formato, como también el derecho de poder conectarse en línea con otros mediante consentimiento de ambos; ser propietarias de su información, compartiéndola cuando deciden y con quienes así lo hacen; acceder libremente a la información por otros puesta a disposición, contando con herramientas prácticas para que ese acceso resulte rápido, fácil y eficaz; contando con derechos y responsabilidades aplicado a todos los usuarios por igual; deben poder forjarse confianza y reputación a través de su identidad y sus conexiones, ser capaces de utilizar Facebook de forma gratuita para establecer presencia, conectarse con otros y compartir información con ellos; Facebook debe publicar su propósito, sus planes y políticas, para fomentar el debate y la participación, debe también traspasar las barreras nacionales y geográficas y estar disponible para todo el mundo, como 'un mundo'." (http:// www.facebook.com/facebook\#!/facebook?sk=info0. Acceso el 4/02/2004)

La comunicación entre usuarios se propicia de diversas maneras: se puede señalar que lo posteado por otros me gusta, puedo comentarlo y compartirlo, en este último caso se trata de trasladar lo publicado por otro usuario al muro propio, el cual es un espacio personalizado donde puede verse la información que cada cual elige postear. El muro funciona como carta de presentación en tanto habitante de este espacio virtual, es la posibilidad de construir un yo virtual, sabiendo que la presentación de sí mismo puede armarse y desarmarse conforme a lo que a cada cual le interese hacer público. Es posible pensar ese tipo de socialización virtual partiendo de la construcción de una versión de sí mismo, seleccionado qué contar y cómo, teniendo como opción compartir esa información o no con los otros usuarios. 
La propia existencia en términos de usuario le lleva a uno a elegir desde dónde definirse y enunciarse ante el mundo y ante los otros integrantes de la plataforma. La posibilidad de compartir posicionamientos en cuanto a gustos, prácticas, elecciones personales -como creencias, relaciones sentimentales, ideologías, política, religión-, nos da una clave para abordar posibles espacios donde se visibilizan relaciones sociales de arraigo, las que actúan como amarras tendidas con otros en tanto sentidos de pertenencia a un lugar (Soraire, 2011).

Ante estas posibilidades, el boom mundial de Facebook cobra dimensiones relevantes para el caso argentino. De acuerdo con las estadísticas proporcionadas por comScore sobre los perfiles de usuarios en Internet de agosto de 2013, los argentinos pasan en promedio cerca de 22 horas mensuales online, ubicándose en la región sólo detrás de Brasil. Respecto al consumo específico de redes sociales, Argentina se destaca por estar tercero a nivel mundial en la cantidad de tiempo dedicado a estas experiencias digitales con nueve horas promedio online, siendo el caso de Facebook el de mayor interés porque el $94 \%$ del consumo se dedica a esta plataforma ${ }^{3}$. La utilización de esta red social no sólo permite interactuar con otros usuarios desde un perfil individual sino también posibilita construir y formar parte de grupos o comunidades donde se comparten intereses, prácticas y se construyen y disputan sentidos.

Al indagar en esta red social, encontramos la presencia de un grupo llamado Infancia en Campamento Vespucio. La característica principal de este grupo es que la mayor parte de sus miembros viven o vivían en el campamento petrolero. Ese espacio es utilizado para compartir fotografías, experiencias y anécdotas, ponerse en contacto con antiguos vecinos, compañeros de escuela, compañeros de trabajo, etc. En la tesis doctoral que utilizamos de punto de partida para la investigación, este grupo se utilizó como fuente para complejizar el análisis acerca de la vida en las comunidades de fábrica ${ }^{4}$.

\section{Campamento Vespucio en Facebook. De comunidad virtual a campo problematizado}

El proceso de enajenación de activos públicos de Yacimientos Petrolíferos Fiscales tuvo como contrapartida la pérdida del empleo para miles de trabajadores que tuvieron que optar, en muchos casos, por abandonar las comunidades que se habían constituido alrededor de los pozos petroleros, y en las que vivieron desde que YPF los empleó. Estas comunidades de fábrica suponían la conformación de villas obreras en las cuales la empresa tenía injerencia no sólo en la producción sino también la reproducción de los trabajadores. Para fijar la mano de obra al territorio, los empleados y sus familias eran beneficiados con la entrega de una vivienda y, además, se creaban las condiciones de infraestructura y servicios que permitían satisfacer las necesidades básicas de la población petrolera. Este proceso, que se inicia en las primeras décadas del siglo XX, y está estrechamente asociado a las políticas empresariales de la YPF estatal, se quiebra con la privatización de la empresa durante la década del noventa (Capogrossi 2013). La consecuencia de ello es una ruptura de los vínculos que los ypefianos habían construido a lo largo de los años, pues gran parte de la población de la villa obrera tuvo que migrar en busca de nuevas oportunidades laborales. Esto supuso la pérdida de lazos con vecinos, compañeros de trabajo, de escuela, amigos de la infancia, etc. Con el paso de los años, y producto del desarrollo de las redes sociales, estas personas encontraron

\footnotetext{
3 Estas estadísticas pueden encontrarse en: http://www.comscore.com/lat/Insights/Presentations_and_Whitepapers/2013/2013_Argentina_Digital_Future_in_Focus

4 En los campamentos petroleros, como Vespucio, se constituyen a lo largo del tiempo lo que se conoce como comunidades de fábrica. Esto supone que la empresa no sólo tiene injerencia sobre la esfera productiva sino también en la reproducción de los obreros. Esto se produce porque no sólo se otorgan las viviendas a los trabajadores -con lo cual se inmoviliza la fuerza de trabajo- si también la firma pone en marcha escuelas, proveedurías, clubes sociales, cines, hospitales, etc. Se crea un complejo entramado que da particularidades a las relaciones que se establecen entre la empresa y sus empleados (Capogrossi 2012)
} 
la manera de reconstituir esos lazos perdidos a través de las tecnologías mediadas por ordenador y, particularmente, recurriendo a un recurso provisto por la red social Facebook. Ese instrumento es un grupo creado al interior de la red denominado Infancia en Campamento Vespucio. .

Las publicaciones en el muro del grupo se estructuran en torno a relatos de acontecimientos, a recuerdos de personajes y lugares vinculados a la cotidianeidad que alguna vez compartieron, a la recuperación de experiencias vividas en el campamento petrolero, a la remembranza de actividades y prácticas que se llevaban adelante en Vespucio o que impulsaba YPF en la región. Esta puesta en común se realiza no sólo a través de relatos en primera persona sino también mediante la publicación de fotografías o vídeos que retratan instantes de estas vidas que interactuaron bajo el ala de Yacimientos Petrolíferos Fiscales. Las interacciones entre los miembros del grupo exceden lo virtual pues, a partir de este recurso técnico, se producen reencuentros cara a cara y se organizan eventos que los nuclean.

Paralelamente, consideramos que Infancia en Campamento Vespucio es utilizado como espacio donde la comunidad virtual ypefiana de Vespucio recrea continuamente sentidos acerca de lo que fue la YPF estatal y la vida dentro de un campamento petrolero. Este dato no es menor pues habla de cierto rol asumido por los ex ypefianos en los procesos de construcción de memorias una vez que fue privatizada la empresa. El corolario del proceso de enajenación de activos públicos petroleros fue la fractura de la familia ypefiana, al expulsarlos de la comunidad de fábrica. Esto hizo que "(...) los [ex] operarios pasen paradójicamente a la posición de herederos simbólicos y culturales de un patrimonio de la fábrica (...)" (Leite Lopes, 1988: 67). Con esto estamos haciendo referencia a que, ante la desaparición del actor que encarnaba, que garantizaba, la existencia de ese relato fundante de la historia petrolera en el norte argentino, los trabajadores asumen la función de guardianes de ese relato. Tomar la historia oficial y hacerla parte del juego, he aquí una de las maneras en que los actores disputan las memorias. Se construye así una suerte de caleidoscopio, donde los cambios en el contexto van creando nuevas configuraciones identitarias en torno a lo "que éramos" y a lo "que somos", en torno al "nosotros" que permite aferrarse a la propia historia cuando el entorno que le daba sustento desaparece.

Este grupo de Facebook de alguna manera encaró ese rol que supone disputar sentidos frente al discurso neoliberal hegemónico de los noventa, que sostenía que las empresas públicas eran deficitarias y generaban un "costo social permanente" precisamente por el entramado socioeconómico, cultural, educativo y recreacional que generaba en el entorno donde se radicaban. A esa construcción discursiva, los ex trabajadores que participan de esta comunidad virtual, le contraponen otros sentidos donde prima la idea de gran familia, de espacio idealizado donde no existían los conflictos ${ }^{5}$.

Nuestras reflexiones respecto a lo dicho hasta aquí nos llevan a preguntarnos acerca de un espacio donde lo instantáneo y masivo que el mundo contemporáneo impone se materializa en un compartir con otros que formaron parte de un mismo colectivo de trabajo. En ese espacio es posible abordar aquellas amarras tendidas hacia una delimitación históricamente específica, que nos lleva atrás en el tiempo y nos remite a un lugar puntual y a su construcción histórica como tal: el Campamento Vespucio, en la provincia de Salta, donde habitaban los trabajadores de YPF con sus familias, hasta la privatización de la década de 1990.

Años han pasado desde entonces, pero no por eso el campamento ha caído en el olvido de quienes lo habitaron. Aunque vivan ahora en distantes y distintos lugares, las relaciones sociales fruto de esa convivencia regresan al presente para ser reconstruidas y reconstituidas

5 Como sostiene Neiburg: “(...) la producción de la categoría de «gran familia» implica la reproducción de sus contenidos, la aceptación de que ella supone un «clima moral», sellado por la confianza y la seguridad de obtener «beneficios», y que exige el respeto de las reglas de conducta que legitiman (...) el mecanismo por el cual se efe efectúan las solicitudes (...)" (Neiburg, 1988) 
en los espacios virtuales, como lo es un grupo en/de Facebook. Nos percatamos entonces de la importancia fundante que las relaciones de trabajo tienen en la vida de las personas, en los sentidos de pertenencia que se entrelazan con el correr del tiempo y en las disputas de sentido acerca de lo que fue y es YPF, siendo en esto clave el lugar físico donde esas relaciones se enmarcan, en este caso, el campamento petrolero.

Hay diversos procesos de construcción socio/política vinculados con el hecho de que los lugares son creados, imaginados, contestados, pero también algunas veces impuestos (Gupta; Ferguson, 1997). Si pensamos que el lugar donde se trabaja es también un lugar que se habita, todo lo que allí se comparte con otros a lo largo del tiempo se vuelve patrimonio de un nosotros determinado, se generan relaciones afectivas para con el lugar y arraigos que soportan el paso del tiempo.

No está de más aclarar que el trabajo es una de las prácticas que organizan la existencia y dan sentido a la vida. El modo de hacer y pensar lo que se hace, cual eje medular donde se despliega la subjetividad y la construcción de la identidad, remite a tener presente tanto las transformaciones sobre la subjetividad y la vivencia del trabajo, como la representación que el trabajador se hace de sí, desde la construcción de su proyecto de vida (Wallace, 1997).

Cuando ese nosotros se ve modificado por las transformaciones que el mercado de trabajo instala, la construcción identitaria asociada a ese colectivo se desplaza hacia otros espacios que facilitan, al albergarla, la continuidad de su dimensión compartida. Así, el nosotros, compañeros de trabajo, por ejemplo, puede visibilizarse aún entre personas que ya no comparten el mismo espacio laboral y de vida, pero que sí lo han hecho durante años.

Los espacios virtuales propician este tipo de encuentros entre actores que en algún momento de su vida han compartido un lugar de trabajo y que en el presente ya no lo hacen. Esto no significa, de ninguna manera, que dejen de identificarse con el colectivo de trabajadores que por tantos años los cobijó, como en el caso de los ex ypefianos. El lugar de trabajo y residencia, en este grupo, atraviesa la primera noción de comunidad que se enuncia de modo virtual en Infancia en Vespucio. El primer centro de referencia de la comunidad es el lugar físico/geográfico que se habita -o se habitaba-, y que se recrea al compartir experiencias en Facebook, con otros que han estado allí también presentes.

Consideramos que pueden señalarse los arraigos territoriales de las personas que construyen usuarios en Facebook a partir de lo que allí cuentan. El territorio, expuesto en espacios virtuales y visto como propulsor de la idea de comunidad, puede que nos esté diciendo algo sobre aquel núcleo de personas que estuvo arraigado en un espacio físico particular y que se ha reencontrado ahora en un espacio virtual o, en otras palabras, que se ha vuelto a reagrupar en una comunidad, esta vez virtual.

Pensamos que en los abordajes metodológicos propios de investigaciones en ciencias sociales, la consulta a espacios virtuales como Facebook es parte de las condiciones materiales cotidianas en las que producimos investigación y, teniendo en cuenta que las amarras para con el territorio no sólo son legítimas cuando trazan límites desde este río hasta ese árbol, sino también desde el nosotros que allí habitaba y vivía en comunidad, no podemos entonces evitar preguntarnos: ¿Cómo dar cuenta de la complejización reflexiva que las posibilidades y limitaciones del análisis en espacios virtuales ha sumado al trabajo que realizamos? En la búsqueda de estas respuestas, nos volcamos a desentrañar lo que damos en llamar comunidades virtuales.

\section{Las tramas de la virtualidad...}

Las primeras preguntas que uno realiza cuando se dispone a abordar Internet en los estudios sociales son ¿cuál es su contexto de surgimiento? Y a partir de eso, ¿qué es la virtualidad? ¿De qué manera esta tiene relación con distintos aspectos de la vida social? ¿Qué la diferencia con lo real? ¿O es un aspecto de lo real? Una serie de autores han trabajado estos 
temas desde diferentes dimensiones: Gustavo Lins Ribeiro (2002), Manuel Castells (2001), Christine Hine (2006), por nombrar algunos, han intentado sistematizar estas problemáticas. Aquí tomaremos sólo algunas categorías que tienen vinculación con nuestro trabajo, entre ellas, las nociones de virtualidad, comunidad y red son fundamentales.

Manuel Castells en su libro La Galaxia Internet (2001) sostiene que, así como la electricidad contribuyó al desarrollo de la fábrica y la gran empresa -pilares organizativos de la sociedad industrial-, Internet constituye en la actualidad la base tecnológica sobre la cual se asienta la era de la información. La red sería su forma organizativa y a ésta la define como "un conjunto de nodos interconectados", que si bien existen como formas muy antiguas de la humanidad, actualmente han sido redefinidas al convertirse en "redes de información" impulsadas por Internet. Esa nueva estructura social basada predominantemente en las redes, es consecuencia de la confluencia de tres procesos independientes entre sí: 1) la necesidad de la economía de flexibilidad en la gestión y globalización del capital, la producción y el comercio; 2) las demandas de una sociedad donde la libertad individual y la comunicación abierta se convirtieron en fundamentales; 3 ) los avances en la informática y las telecomunicaciones derivados de la revolución de la microelectrónica. Esos tres procesos posibilitaron la constitución de una "sociedad red", donde las principales actividades económicas, sociales, políticas y culturales se estructuran a través de Internet (Castells, 2001:15-16) ${ }^{6}$.

Este autor sostiene que la comunicación consciente (el lenguaje) es lo que determina la especificidad biológica de la especie humana, por ello “... como la actividad humana está basada en la comunicación e Internet transforma el modo en que nos comunicamos, nuestras vidas se ven profundamente afectadas por esta nueva tecnología de la comunicación. Por otro lado, al utilizar internet para múltiples tareas, vamos transformándola. De esta interacción surge un nuevo modelo socio-técnico" (Castells, 2001: 19).

En el mismo sentido se expresa Gustavo Lins Ribeiro en su artículo "El Espacio Público Virtual". Allí sostiene que la virtualidad es una entidad compleja que participa de diversas maneras en la vida social y psicológica de las personas. Si bien, desde el sentido común, uno vincularía este término con la mundialización de la Internet en 1995 y la participación de los sujetos en el ciberespacio, él separa esta noción de la de tecnología. Para el autor, la virtualidad existe con anterioridad a la incorporación de nuevas tecnologías de la comunicación porque considera que la sensibilidad a la virtualidad es una característica humana general. Esa sensibilidad se hallaría imbricada al uso del lenguaje en tanto somos sujetos capaces de imaginar lo que no está, de crear estructuras que son abstracciones antes de tornarse hechos empíricos ${ }^{7}$. Una de las funciones de la virtualidad en la que hace hincapié Lins Ribeiro es aquella que permite constituir sujetos colectivos:

"pues es la capacidad de virtualizar que nos asegura, a través del lenguaje, nuestra participación en totalidades sociales más amplias que las que aquellas que fenomenológicamente experimentamos. Como los signos y sistemas simbólicos son la matriz de la que deriva la virtualidad, así como son imaginadas, todas las comunidades son virtuales, en el sentido de que no

6 La noción de "sociedad red" ha sido objeto de múltiples debates. Aquí no nos vamos a centrar en ellos porque no es el tema que nos ocupa. Si nos interesa tomar este concepto para situar el problema que analizaremos en un contexto macro.

7 Lo virtual ha sido un concepto muy desarrollado en los estudios de Pierre Levy, quien lo define no como una oposición a lo real sino como "una forma de ser fecunda y potente que favorece los procesos de .creación, abre horizontes, cava pozos llenos de sentido bajo la superficialidad de la presencia física inmediata". (Levy, 1999: 8) Este autor, también señala que en el libro Atlas de Michel Serres se ilustra el tema de lo virtual, y nos recuerda que 'La imaginación, la memoria, el conocimiento y la religión son vectores de virtualización que nos han hecho abandonar el «ahí» mucho antes que la informatización y las redes digitales.” (Ídem:14) 
pueden ser abarcadas en su totalidad por un individuo y en el sentido de que existen, en la mayor parte del tiempo, como potencialidad y no como realidad, simulando la existencia de un sujeto colectivo. Las comunidades imaginadasvirtuales son construidas por intermedio de sistemas simbólicos que pueden tener por soporte técnicas sociales, como los rituales, o aparatos técnicos, sobre todo los vinculados a la (re)producción de información (signos e imágenes) y a la comunicación" (Lins Ribeiro, 2002: 2).

Como nos interesa pensar en las redes sociales como fuente, no podemos abstraernos de este aspecto, de esta pregunta primigenia porque, de acuerdo a cómo nos situemos frente a la virtualidad, podremos problematizar de una manera u otra nuestro abordaje teóricometodológico. El hecho de considerar la virtualidad como un aspecto más de la vida de los sujetos, permite pensar en la tecnología y en los usos que los sujetos hacen de ella. Y al pensarlo desde esta perspectiva, la construcción de espacios de interacción utilizando Internet se vuelve plausible de ser objeto de análisis porque de otro modo, estaríamos frente a un universo ficcional donde nada sería fiable ni auténtico. Situarse frente a la virtualidad como un aspecto más de la vida social del hombre, es lo que posibilita el entrecruzamiento de lo offline y lo online, enriqueciendo las posibilidades de análisis porque de esta manera podemos observar cómo los sujetos construyen prácticas, discursos y recrean experiencias utilizando las nuevas tecnologías.

\section{Comunidad (es...)}

Varios autores sostienen que la utilización de Internet en las investigaciones sociales, particularmente en la antropología, supone redefinir ciertos conceptos y así adaptarlos a un nuevo contexto mediado por tecnologías de la comunicación. Contexto donde los modos de abordar el objeto de estudio supone establecer ciertas pautas de análisis o realizar ciertas preguntas que permitirían pensar lo digital como fuente y como problema de investigación. Entre esos autores, Hine reflexiona acerca de las maneras de abordar Internet situándose desde la etnografía. Por un lado, encuentra que se puede definir a Internet como espacio donde se gesta cultura, en ese sentido, los análisis sobre ella permitirían observar de qué maneras la utilizan diferentes actores sociales. Así mismo, se estaría refiriendo a la constitución de una comunidad de prácticas de conocimiento, lenguaje y bienes compartidos (Hine, 2006: 43). Esta dimensión, se asienta en una nueva definición de comunidad sustentada más en prácticas compartidas, que en condiciones físicas. Lo que intenta la autora es poner en cuestión la idea de "presencialidad" en la etnografía porque en la actualidad la posibilidad de interacciones mediadas por tecnologías permiten repensar el rol de la presencia física como fundamento de la etnografía (Hine, 2006: 59).

Por otro lado, Internet podría definirse cómo artefacto cultural, esto es, como tecnología creada por personas concretas, para fines concretos y en un contexto particular. En ese sentido define a la tecnología como,

"resultado de procesos de negociación entre distintos grupos de interés que observan en ella ventajas y desventajas. El resultado de estos procesos implica un juego de fuerzas entre varias definiciones de tecnología y una versión final que refleja de algún modo las aportaciones de los grupos sociales relevantes" (Hine, 2006:46).

La relación entre lo social y la técnica es fundamental para Christine Hine pues es lo que permite utilizar Internet en los estudios etnográficos. Lo que ella sostiene es que para pensar Internet desde estos dos ámbitos debe traspasarse el límite entre lo offline y lo online y, para 
ello “(...) es necesario repensar la relación entre espacio y etnografía” (Hine, 2006: 18-19).

La redefinición de esa relación que menciona la autora pasa por la puesta en tensión de la presencia física del investigador en un espacio determinado. En este sentido lo que proponen algunos investigadores es la realización de una etnografía multisituada que trascienda la necesidad de ubicar el trabajo etnográfico en un territorio delimitado y “(...) permita al etnógrafo seguir a la gente, a los artefactos, a las metáforas, historias o conflictos allí donde se produzcan sin quedar atado a la necesidad de mantener su unidad de análisis en una determinada zona geográfica o en un determinado contexto" (Ardèvol; y otros, 2003: 74).

Esta puesta en cuestión de los parámetros tradicionales de la etnografía -que tiene por detrás un debate extenso y que abarca la última década-, supone también la redefinición de categorías que han sido muy caras para la antropología (Beaulieu, 2004. Domínguez, 2007. Escobar, 2005. Estalella; Ardevol, 2007. Hine, 2004 [2000]. Kozinets, 2010. Mayans, 2002. Miller y Slater, 2000. Paech, 2009. Sade-Beck, 2004, entre otros). Entre ellas, el concepto de comunidad es fundamental y necesita ser rediscutido. Tradicionalmente esta categoría se hallaba asociada a ciertos parámetros espacio-temporales, que incluían la territorialidad y un período delimitado de tiempo. Pero con el tiempo y con la incorporación de otras dimensiones de análisis, la noción de comunidad dejó de estar asentada en esas características, para incluir prácticas, discursos y experiencias compartidas, un sentido de pertenencia que va más allá de la proximidad física. (Heiss, 2007. Mosquera Villegas, 2008. Preece; y otros, 2003. Wellman; y otros, 2002, entre otros)

En esa dirección se orienta la noción de comunidades virtuales que emerge en los primeros estudios acerca de la red. En 1995 este término es acuñado por Howard Rheingold quien lo define como "agregaciones sociales que emergen de la red cuando un número suficiente de personas entabla discusiones públicas durante un tiempo lo suficientemente largo, con suficiente sentido humano, para formar redes de relaciones personales en el ciberespacio" (Rheingold, 1996:20). A partir de entonces distintos autores se preocuparon por dar mayor especificidad a este concepto y así encontrar un modo de definir las diferentes interacciones que se producen a partir de la utilización de la red (Wellman; Gulia, 1999. Smith; Kollock, 1999. Johnson, 2001, entre otros). Aquí tomaremos la definición construida por Magallanes Udovicich, quien sostiene que una comunidad virtual es:

"un conjunto de personas que se agrupan en el ciberespacio en torno a intereses comunes y que establecen vínculos entre ellos, utilizando como medio de comunicación y relación las herramientas hipermediales que brinda la Internet. En nuestra definición (...) tenemos en cuenta las características que Ontalba (2002) reconoce como habituales en las distintas definiciones de este concepto: a) son agrupaciones de personas, b) reunidas en torno a intereses comunes, y c) que utilizan la Red como medio de relación" (Magallanes Udovicich, 2010: 3).

El eje en torno al cual gira esta conceptualización es que el desarrollo de nuevas tecnologías de comunicación produce mutaciones en las maneras en que los sujetos interactúan, con lo cual nos vemos obligados a redefinir o rediscutir algunas categorías. Como sostiene esta autora:

"Hoy sabemos que la noción del espacio como territorio ha perdido estatus conceptual al aparecer en escena el ciberespacio. Esto implica que, en el espacio virtual, se borran las limitaciones territoriales al interactuar con el mundo superando las fronteras geográficas sin moverse del lugar de origen. Asimismo, reconocemos que el tiempo ha adquirido nuevas dimensiones a través de su anclaje en la experiencia digital, donde las interacciones online pueden ocurrir en alta velocidad y simultaneidad. El tiempo virtual del ciberespacio 
logra traspasar el tiempo real con comunicaciones sincrónicas y asincrónicas gracias al uso de las nuevas tecnologías de la información y la comunicación. Puesto que estas transformaciones repercuten directamente en los procesos de socialización del hombre, no es menor considerar su impacto en la forma de concebir sus comunidades de pertenencia”. (Magallanes Udovicich, 2010: 2)

La puesta en cuestión de las ideas de tiempo y espacio a la hora de definir una comunidad virtual permitiría cruzar una y otra vez los límites entre lo offline y lo online posibilitando enriquecer el universo de información al que el investigador accede. Porque uno de los interrogantes que surgen cuando pensamos en la posibilidad de cruzar datos entre ambas dimensiones es ¿Qué cosas los sujetos muestran o escriben en el universo online? ¿Son las mismas que aparecen cuando la interacción es cara a cara?. En el caso que nosotros analizamos pudimos descubrir que las diferencias entre lo que se decía en las entrevistas y lo que se publicaba en el grupo de Facebook eran importantes. A la hora de encontrar una explicación a ello, quizás sea de utilidad detenerse un momento en las características de esta red social y de su utilización como fuente, haciendo hincapié en las potencialidades y limitaciones que posee.

\section{Las redes sociales como fuente: el caso de Facebook}

En este artículo, sostuvimos precedentemente, nos vamos a detener en la utilización de Facebook como fuente en las investigaciones en ciencias sociales, particularmente en los estudios de carácter etnográfico. Pero primero necesitamos señalar qué entendemos como fuente. En primer lugar podemos hacer referencia a todo aquel material que nos brinda información acerca de un problema de estudio. En ese sentido, las fuentes pueden ser de dos tipos: primarias y secundarias o literatura crítica. Las primarias son aquellas que nos proporcionan datos de primera mano acerca del objeto que investigamos y han sido producidas por los actores involucrados o en el período de tiempo analizado. Entre ellas podemos encontrar distintos tipos de documentos, entrevistas, cuestionarios, literatura, noticias, fotografías, obras de arte, cartas, etc (Eco, 2004: 65-71). La selección de una fuente de este tipo estará estrechamente vinculada al problema que se aborde. La multiplicación de tecnologías de la información en las últimas décadas no sólo ha facilitado y ampliado el acceso a ellas sino también ha posibilitado incorporar nuevos formatos a esta categoría. El caso que aquí analizamos fue abordado como una fuente de este tipo. Las fuentes secundarias, por otro lado, son todas aquellas producciones que analizan e interpretan las fuentes primarias. Entre ellas podemos nombrar artículos de revistas, libros de texto, enciclopedias, biografías, etc.

Como señalamos más arriba, el desarrollo de nuevas tecnologías de la comunicación ha multiplicado el espectro de fuentes que se pueden utilizar en las investigaciones. En el caso que hemos analizado, la red social Facebook nos permitió observar la conformación de una comunidad virtual formada por un colectivo de personas que vivieron en un mismo espacio, un campamento petrolero, el cual inicia su proceso de fragmentación en la década del noventa cuando se privatiza la empresa estatal Yacimientos Petrolíferos Fiscales.

\section{La posicionalidad como tensión etnográfica}

La posibilidad de incorporar nuevas fuentes, como las redes sociales en nuestra investigación, fue posible porque nos situamos en una perspectiva que considera que todo proceso de investigación tiene algo de artesanal puesto que la versión final no es producto de una receta pautada de antemano, sino que el mismo es resultado de la biografía particular de cada investigador, de las opciones teóricas que se adopten, de la confrontación de las categorías utilizadas con la experiencia de contextos socio-históricos particulares y de situaciones imprevisibles que se presentan en la cotidianeidad del trabajo de campo (Peirano, 
2004: 22). De esta manera, se abren nuevos senderos por dónde deslizar la investigación y es posible establecer un diálogo tridimensional: con los informantes, con la(s) historia(s) disciplinar(es) desde las cuales uno se posicione y con el azar.

La red social Facebook, como sostuvimos, permite a los individuos crear perfiles propios y a la vez grupos con intereses compartidos. En el caso que analizamos, tomamos uno de esos grupos cuyo nombre es "Infancia en Campamento Vespucio". Lo que nuclea a casi todos sus miembros es residir o haber residido en esa localidad del noroeste de Salta y haber sido trabajadores o familiares de trabajadores de Yacimientos Petrolíferos Fiscales. En la actualidad, el grupo cuenta con 1.592 miembros que comparten a través del muro recuerdos, fotografías e impresiones acerca de lo que fue su vida en el campamento petrolero.

Nuestro proceso de acercamiento al grupo constó de varias etapas. La primera de ellas implicó la observación y el registro etnográfico de lo que sus miembros hacían público en el muro. Es necesario señalar que este grupo es de carácter abierto, lo que quiere decir que cualquier persona que lo encuentre tiene acceso a la información que allí se publica ${ }^{9}$. Lo que allí encontramos fue sistematizado y, luego de evaluar si lo que se compartía en el grupo podía ser utilizado como fuente de primera mano en nuestras investigaciones, decidimos pedir la incorporación como miembros y enviar un mensaje privado a la administradora. En ese mensaje nos presentamos, contamos cuáles eran los objetivos de la investigación y enviamos nuestro correo electrónico para que todos aquellos que quisieran ponerse en contacto pudieran hacerlo. A partir de entonces comenzó un intercambio de e-mails con distintos miembros de esta comunidad virtual donde ellos preguntaban quiénes éramos, por qué estábamos interesados en las personas que habían trabajado y/o residido en Campamento Vespucio. Todas las dudas que surgieron fueron contestadas y se les consultó si estaban dispuestos a realizar un cuestionario de veinte preguntas. De los quince enviados, respondieron la mitad. Con otros, pudimos encontrarnos sin mediaciones digitales y realizar entrevistas.

Más allá de que una herramienta online nos permitió entrar en contacto con informantes y entrevistarlos, también queremos reseñar que hemos realizado una observación de campo del muro del grupo y sistematizado las maneras en que sus miembros lo utilizan. En ese orden de cosas pudimos identificar tres maneras de participar a través de la red social: 1) como vehículo de memorias; 2) como espacio que posibilita recuperar antiguos vínculos; 3) como espacio de disputa de sentidos acerca de lo que fue YPF.

En el primer caso, partimos de una definición de memorias de Elizabeth Jelin quien sostiene que estas son construcciones sociales que se redefinen una y otra vez en el marco de una relación con el otro. En ese sentido los sujetos seleccionan hitos, acontecimientos que suponen negociaciones y disputas de sentidos del pasado y que encuadran la memoria (Jelin, 2001: 7-9). La noción de experiencia adquiere centralidad aquí en tanto es vivida subjetivamente y es culturalmente compartida y compartible porque "Es la agencia humana la que activa el pasado, corporeizado en los contenidos culturales (...). La memoria, entonces, se produce en tanto hay sujetos que comparten una cultura, en tanto hay agentes sociales que intentan «materializar» estos sentidos del pasado en diversos productos culturales que son concebidos como o que se convierten en vehículos de memoria, tales como libros, museos, monumentos, películas o libros de historia" (Jelín, 2001:17).

Si bien esta autora no incorpora formatos digitales en su definición, a nosotros nos parece que dada la utilización que los miembros del grupo de Facebook hacen, este podría ser

8 Cabe destacar que al momento de iniciar el abordaje de este grupo, el mismo contaba con menos de 500 miembros. En tres años, el número se ha triplicado. Posiblemente esto se deba a la expropiación de la mitad de las acciones de Repsol-YPF S.A. por parte del gobierno argentino durante los primeros meses de 2012.

9 Los grupos que se abren en Facebook pueden ser abiertos como describimos, cerrados (todo el mundo puede verlo pero sólo se puede ingresar y participar si un administrador lo autoriza), secretos (sólo pueden verlos aquellas personas que han sido invitadas a formar parte de él). 
pensado como vehículo de la memoria ${ }^{10}$. Porque si hay algo que lo caracteriza es la necesidad de recuperar y construir memorias acerca de una etapa de la vida de sus miembros, etapa marcada por la residencia en Campamento Vespucio y por la injerencia de la petrolera estatal no sólo en la esfera de la producción sino también en la de la reproducción de los trabajadores petroleros y sus familias.

En este sentido, nos interesa resaltar la tensión etnográfica que se nos presentó en torno a estos espacios, en torno a la posicionalidad del investigador. En primer lugar, el uso de Facebook forma parte de nuestra vida cotidiana ya que como usuarias integramos esta red social, la dinámica de encuentro en la misma no nos es ajena y es una de las posibilidades existentes a la hora de tomar contacto con las personas o grupos con los que decidimos relacionarnos, tanto en lo que hace a nuestra vida privada, como así también a la práctica profesional que ejercemos, la investigación.

Abu Lughod (2006) señala que tanto la posicionalidad del sujeto como las representaciones que este hace de otros, son verdades posicionadas que sirven para distanciarlo de los demás. Son una herramienta para hacer al otro, donde hay un lenguaje de poder en los que creen ubicarse afuera y aparte de lo que describen. El afuera sería una posición dentro de un complejo político e histórico que jerarquiza y distingue un "ellos" y congela las diferencias, como fronteras fijas, estables y coherentes. De este modo, estudiar la propia sociedad acarrearía el problema de obtener distancia, la necesidad de mantenerse aparte del otro. Mencionar en los escritos etnográficos lo que nos permite estudiar lo que estudiamos, es una estrategia ante el hecho de que no se puede escapar a la posicionalidad.

Hemos construido nuestros "usuarios" brindando cierta información sobre nosotras mismas, quedando así en evidencia los lazos de pertenencia desde los cuales nos definimos ante el mundo. Lo que compartimos -mediante los posteos, los me gusta, etc- nos revela ante los demás usuarios, nos posiciona de cierto modo. Esta observación se complejiza ante el hecho de que la virtualidad nos permite prescindir de la presencia física -tan preciada por la antropología clásica de corte europeo en la consecución de las etnografías-, para entablar cualquier diálogo. En este punto vale recalcar que el posicionamiento va más allá de ella en el trabajo de campo y en esta ocasión en concreto, incluso se vuelve prescindible en el tejido artesanal que trama el devenir de la investigación. Podemos valernos de este espacio virtual para estar presentes en diferentes lugares y momentos, dejando huella del posicionamiento desde el cual lo hacemos.

En sintonía con ello, notamos también que, si bien se da por sentada la ausencia del "territorio" en las interacciones en Facebook -como espacio que rompe la diada espacio/ tiempo al posibilitar el diálogo en todo momento- esto no significa que desaparezca la noción de "lugar". Consideramos que es esa noción la que nos permite identificar las amarras que aglutinan a este tipo de comunidades virtuales. En el caso que nos ocupa, las más claras serían el lugar de trabajo y la estructuración de la vida en el campamento. Con esto quisiéramos señalar que puede que la red haya logrado prescindir del lugar físico como punto fundante de lo que ella es, pero no por eso, el territorio está ausente. Por el contrario, éste emerge en el contenido de lo que se comparte, en lo que termina dando sentido al grupo.

Consideramos que desterritorializar los lugares dándoles solo sentido de espacio, es una apuesta política propia del neoliberalismo, apuesta que apaña la privatización como ejercicio de apropiación de territorios devenidos productivos, vaciados así de historicidad.

El trabajo etnográfico realizado atravesó la tensión generada por dos elementos: la posicionalidad del investigador y la desterritorializacion del campo, ambos inherentes al uso

10 Una posible aproximación, en este sentido, implica considerar Infancia en Campamento Vespucio actuando como memoria digital social de tipo comunitaria-territorial, en tanto el lugar de los intereses compartidos de la comunidad virtual, donde se nuclean afectos, tradiciones y valores, giran en torno a una fuerte base geográfica/ geo-simbólica compartida. (García Gutiérrez, 2005, citado por García Jiménez ;y otros, 2010) 
hecho de un espacio virtual como el señalado. En este proceso, una de las primeras preguntas que nos hicimos al hallar a este grupo de Facebook fue de carácter ético, porque nos encontramos observando una comunidad virtual sin que los miembros de la misma tuvieran conocimiento de lo que hacíamos. Quizás este sea el problema principal que emerge cuando uno pone en tensión la presencialidad física del investigador en el campo ${ }^{11}$.

A esto lo resolvimos uniéndonos a este grupo y enviando un mensaje a la administradora que luego circuló entre todas las personas que pertenecían a "Infancia en Campamento Vespucio", más allá de que las políticas de Facebook ponen en aviso a los usuarios que todo lo que compartan puede ser utilizado por otros usuarios, con fines personales, sin que implique esto la necesidad de un permiso previo.

Asimismo, de igual manera que Beaulieu (2004: 153), cuando plantea el dilema entre el anonimato de los informantes respecto a las necesidades de citación en las publicaciones académicas, nos preguntamos qué hacer con las publicaciones de las personas del grupo que queríamos citar en la investigación. A lo largo del proceso realizamos un registro etnográfico pormenorizado de todo lo que se posteaba en el muro y a esa información la parafraseamos o la incluimos textualmente manteniendo el anonimato de quién la emitía. Pero esto fue un criterio a seguir tanto con este tipo de fuente como con las entrevistas.

Sin duda, otro aspecto ineludible de un proceso de investigación que contemple este tipo de comunidades es el de la mutabilidad de lo que se publica. Quién utiliza Facebook puede editar cuantas veces quiera lo posteado o eliminarlo en cualquier momento. El problema de la mutabilidad del recurso técnico ${ }^{12}$ y de la volatilidad de la información nos hizo reflexionar acerca de la flexibilidad en los modos de registro que debe asumir el investigador. Nosotros no sólo llevamos minuciosamente un cuaderno de campo donde transcribimos todo lo observado, sino también realizamos capturas de pantalla que nos permitieron documentar el muro del grupo.

Para finalizar estas primeras notas que intentan sistematizar de alguna manera el uso de las redes sociales en las investigaciones, quisiéramos señalar la potencialidad que tiene el uso de este tipo de fuentes. Pudimos entrever en una primera aproximación que lo que se expresaba dentro de esta comunidad virtual difería de lo que en las entrevistas era seleccionado por los informantes para relatar. Tal vez esta herramienta que brinda Internet sea un facilitador que impulse a las personas que han vivido la fragmentación de sus marcos de referencia por un proceso privatizador a expresar más abiertamente sentidos, apreciaciones, memorias, experiencias y deseos. La posibilidad que brinda sentarse tras un ordenador y, con tiempo, reflexionar acerca de lo que se quiere decir, compartir o cuestionar, es un aspecto que quizás nos interpele cuando, desde la antropología, repensamos nuestras prácticas.

\section{Consideraciones finales}

A partir de este trabajo reconocemos que el carácter inestable y cambiante de la infor-

11 Dicha reflexión, nos insertó en las discusiones en torno a la posición que asume el investigador en el marco de las etnografías virtuales. La posibilidad de permanecer o no como 'Lurker' (mirón, participante pasivo) emerge en las discusiones de los estudios de/en Internet en tanto elemento ético del etnógrafo en este tipo de campo, distinguiendo grados de anonimato, participación e interacción para/con los usuarios (Sade-Beck, 2004. Paech, 2009). Nosotras hemos reconocido en el devenir de la investigación la potencialidad que el posicionamiento del investigador en el campo mediante la interacción con los demás usuarios como un espacio propicio para una compresión mayor del campo de estudio y las prácticas estudiadas. Por lo tanto, optamos por dotarnos de visibilidad y explicitar el rol del investigador en el campo a partir de intercambios explícitos con los otros usuarios, tal como sugirieron Estalella y Ardevol (2007)

12 Numerosos autores trabajan esta mutabilidad en relación a la rápida obsolescencia de estas tecnologías y su impacto en los estudios antropológicos de las comunidades virtuales, como por ejemplo, Wilson y Peterson (2002) 
mación disponible en entornos online propicia la emergencia de múltiples retos a los investigadores del ciberespacio.

Por un lado, se presentan claros desafíos de accesibilidad a los datos ya que los distintos tipos de grupos permiten la visibilidad o invisibilidad de determinadas informaciones de acuerdo al grado de relación entre los contactos. Asimismo, las gestiones de privacidad permiten a los usuarios regular el contenido que se muestra en los perfiles individuales y grupales en Facebook, lo que se traducirá en que el resto de los usuarios tendrán accesos diferenciados a cada una las informaciones presentadas, incluyendo los investigadores. Por otro lado, una vez que se accede a los datos, no se puede suponer una estabilidad de la información publicada debido a que los contenidos pueden ser eliminados, editados u ocultos en el transcurso de la investigación. De esta manera, se vuelve imprescindible innovar en técnicas de registro de datos que acompañen el proceso de cada publicación, lo que supone la gestión de una cantidad de información considerable. Todos estos elementos van delineando un desafío técnico que, en conjunto con la necesidad del manejo de la tecnología Facebook, delinean un campo de reflexión y análisis de vital importancia para el abordaje de diversos fenómenos en esta red social.

En segundo término, reconocemos un desafío legal acerca del tratamiento y publicación de los datos contenidos en Facebook para la investigación científica. Por más que cada usuario tenga la posibilidad de visibilizar o no el contenido de lo publicado en sus perfiles individuales y los grupos, Facebook presenta una política de privacidad que delimita las posibilidades de acceso y publicación de los datos. Algunos elementos de las políticas de privacidad deben ser tenidos en cuenta en los trabajos de investigación: por un lado, las modificaciones técnicas de presentación de datos y de políticas de privacidad que se gestionan desde la empresa de servicios y que implican actualizaciones o modificaciones en las presentaciones y manejo de datos. En segundo lugar, las cuestiones legales asociadas al manejo de datos, tanto de los derechos como las obligaciones en el manejo de la información publicada y a la que se accede, sobre todo en los casos de mayor nivel de privacidad como el caso de los perfiles cerrados o secretos.

Finalmente, reconocemos el desafío ético. Ya hemos mencionado a lo largo del trabajo, las dificultades para trabajar desde el rol de investigador, donde la presencia explícita y con las autorizaciones correspondientes de los usuarios para trabajar sobre sus perfiles o la información personal o colectiva que publican dentro de estos espacios. En este sentido, reconocemos la importancia de los sucesivos intentos de consensuar guías éticas para el estudio de Internet desarrolladas por la Aoir (Association of Internet Researchers) (Ess, 2002) o la introducción de especificaciones para Internet, realizado por el National Comittee for Research Ethics in the Social Sciences and the Humanities (Elgesem, 2002), ya que son grandes avances en este sentido (Estalella; Ardevol, 2007). Sin embargo, aún resta mucho camino ante la necesidad de proteger las fuentes para el caso de la producción científica en tensión con los reconocimientos necesarios para quienes han publicado información privada de acceso público o semipúblico en estos espacios. La tensión entre accesibilidad y libre uso forma parte entonces de los desafíos con los que se enfrenta el investigador en la plataforma Facebook.

\section{Bibliografía}

ARDEVOL, Elisenda; BERTRAN, Marta; CALLEN, Blanca; PEREZ, Carmen

2003 "Etnografía virtualizada: la observación participante y la entrevista semi-estructurada en línea", en Athenea Digital , 3.

BEAULIEU, Anne

2004 "Mediating Ethnography: objectivity and making of ethnographies of the Internet, en Social Epistemology”, 18, 2-3: 139-163. Rotterdam. http://www.virtualknowledgestudio.nl/ staff/anne-beaulieu/documents/mediating-ethnography.pdf (4/10/2013). 
CAPOGROSSI, María Lorena,

2013 La relación capital-trabajo en «economías de enclave» y sus transformaciones durante el proceso de ajuste estructural en los 90. El caso de los trabajadores de YPF en Campamento Vespucio y General Mosconi, Salta. Tesis doctoral, Facultad de Filosofía y Humanidades, Universidad de Buenos Aires. [Orig. Inédito].

2012 "Algunas dimensiones de la relación entre el capital y el trabajo en un enclave petrolero. El caso de Yacimientos Petrolíferos Fiscales en Argentina", en Gaceta Laboral, 18, 3. Venezuela, Centro de Investigaciones y Estudios Laborales y Disciplinas Afines (CIELDA), Facultad de Ciencias Jurídicas y Políticas de la Universidad del Zulia, Septiembrediciembre de 2012

CASTELLS, Manuel

2001 La galaxia internet. Barcelona: Areté.

DOMINGUEZ FIGAREDO, Daniel

2007 "Sobre la intención de la etnografía virtual”, Teoría de la Educación. 8, 1. http://campus.usal.es/ teoriaeducacion/rev_numero_08_01/n8_01_dominguez_figaredo.pdf $(12 / 02 / 2011)$.

ECO, Umberto

$1991 \quad ¿$ ¿Cómo se hace una tesis?. Técnicas y procedimientos de estudios investigación y escritura. España: Gedisa.

ELGESEM, Dag

2002 "What is special about the ethical issues in online research?", en Ethics and Information Technology, 4, 3: 195-203.

ESS, Charles

2002 Ethical decision-making and Internet research: Recommendations from the AoIR ethics working committee. Association of Internet Researchers (AoIR). http://www.research.utoronto.ca/ethics/pdf/human/nonspecific/Internet\%20Research\%20Ethics.pdf(15/05/2007).

ESCOBAR, Arturo

2005 "Bienvenidos a Cyberia. Notas para una antropología de la cibercultura, Revista de Estudios Sociales, 22, diciembre: 15-35. http://www.unc.edu/ aescobar/text/esp/bienvenido\%20a\%20Cyberia.pdf (17/02/2011)

ESTALELLA, Adolfo y ARDEVOL, Elisenda,

2007 "Ética de campo: hacia una ética situada para la investigación etnográfica de internet". Forum Qualitative Sozialforschung / Forum: Qualitative Social Research, 8,3. Disponible en: http://nbn-resolving.de/urn:nbn:de:0114-fqs070328 (11/11/2013)

GARCÍA JIMENEZ, Antonio; BELTRÁN ORENES, Pilar; NÚÑEZ PUENTE, Sonia

2010 "Una aproximación al concepto de frontera virtual. Identidades y espacios de comunicación”, en Revista Latina de Comunicación Social, 65, P.p. 214-221. La Laguna (Tenerife): Universidad de La Laguna. http://www.revistalatinacs.org/10/art2/894_Madrid/16_Antonio_Garcia_et_al.html (7/05/2010).

GUPTA, Akhil y FERGUSON, James

1997 "Más allá de la cultura: espacio, identidad y la política de la diferencia", en www.cholonautas.edu.pe (20/03/2009)

HEISS, Bettina M.

2007 'The Role of the Term 'Online Community' as Coordinative Production Frame for a Growing Multidisciplinary Knowledge Network of Scholars”. Paper presented at the annual meeting of the International Communication Association, TBA. San Francisco. http://www.allacademic.com/meta/p172891_index.html (11/11/2013)

HINE, Christine

2004 Etnografía virtual. Barcelona: UOC.

JELIN, Elizabeth,

$2001 \quad$ Los trabajos de la memoria. España: Siglo XXI editores.

JOHNSON, Christopher

2001 "A survey of current research on online communities of practice", The Internet and Higher Education, 4, 1, 45-60. http://www.sciencedirect.com/science/article/pii/ S1096751601000471 (20/01/2013). 
KOZINETS, Robert

2010 Netnography: Doing Ethnographic Research Online. SAGE

LEITE LOPES, José Sergio

1988 A tecelagem dos conflitos de classe na "cidade das chaminés, Tesis de Doctorado, Progra-

LEVY, Pierre ma de Pos - graduación en Antropología Social. Río de Janeiro: Museo Nacional.

1999 ¿Qué es lo virtual?. Paidós. Barcelona.

LINS RIBEIRO, Gustavo

2002 "El espacio público virtual", Serie Antropológica, 318. Brasilia: Instituto de Ciencias Sociales. Universidad de Brasilia. http://www.dan.unb.br/images/doc/Serie318empdf.pdf. $(25 / 04 / 2012)$.

LUGHOD, Abu

2006 "La interpretación de la(s) cultura(s) después de la televisión”, Etnografías contemporáneas , 1. Buenos Aires, UNSAM.

MAGALLANES UDOVICICH, Mariana Loreta,

2010 "Identidades y pertenencia en comunidades virtuales: Posibles indicios", en: Actas de las IVX Jornadas de Investigadores en Comunicación "Investigación y participación para el cambio social”. Buenos Aires: Universidad Nacional de Quilmes.

MAYANS, Joan

2002 Género Chat. O cómo la antropología puso un pie en el Ciberespacio. Barcelona: Gedisa.

MILLER, Daniel; SLATER, Don

2000 The Internet. An ethnographic approach. Oxford: Berg.

MOSQUERA VILLEGAS, Manuel Andrés

2008 "De la etnografía antropológica a la etnografía virtual”, en Revista Fermentum, 53,18. Mérida. Venezuela. Septiembre-Diciembre de 2008.

NEIBURG, Federico

1988 Fábrica y Villa Obrera: Historia social y Antropológica de los obreros del cemento, dos tomos. Buenos Aires: Centro Editor de América Latina

PAECH Vennessa

2009 "A method for the times: a meditation on virtual ethnography faults and fortitudes", Nebula, 6, 4. http://www.nobleworld.biz/images/Paech.pdf (17/10/2013).

PEIRANO, Maritza

1995 A favor da etonografia. Río de Janeiro: Relume Dumará

PISCITELLI, Alejandro; ADAIME, Iván; BINDER, Inés, (Comps).

2010 El proyecto Facebook y la posuniversidad. Sistemas operativos sociales y entornos abiertos de aprendizaje. Barcelona: Ariel-Fundación Telefónica.

PREECE, J.; MALONEY-KRICHMAR, D.; ABRAS, C.

2003 "History of Emergence of Online Communities", en Wellman, B., Ed., Encyclopedia of Community. Berkshire Publishing Group, SAGE.

RHEINGOLD, Howard

1996 La comunidad Virtual: Una sociedad sin fronteras. Barcelona: Gedisa Editorial.

SADE-BECK, Liav

2004 "Internet ethnography: Online and offline", en: International Journal of Qualitative Methods, 3, 2. http://www.ualberta.ca/ iiqm/backissues/3_2/pdf/sadebeck.pdf $(3 / 11 / 2013)$.

SMITH, Marc; KOLLOCK, Peter, (eds).

1999 Comunidades en el ciberespacio. Barcelona: UOC. 1. ${ }^{\text {a }}$ ed.

SORAIRE, Florencia

2011 "Que hablen, nosotros laburamos. Mineros y ambientalistas, enfrentamiento vistualizado", XXVIII CONGRESO INTERNACIONAL DE ALAS, 6 a 11 de septiembre de 2011, UFPE, Recife-PE., Brasil.

WALLACE, Santiago

1997 "Trabajo y subjetividad. Las transformaciones en la significación del trabajo", en Actas de la II Jornada de Antropología de la Cuenca del Plata, (Vol. VI). Rosario, Argentina. 
WELLMAN, Barry; GULIA, Milena

1999 "Net-Surfers Don't Ride Alone: Virtual Communities as Communities", en: B. WELLMAN (ed.), Networks in the Global Village: Life in Contemporary Communities. Boulder: Westview Press. 331- 366.

WILSON, Samuel. M. ; PETERSON, Leighton. C.

2002 "The anthropology of online communities", Annual Review of Anthropology, 31: 449-67.

\section{3}

